Article

\title{
Shock Waves Asymmetry in a Symmetric Nozzle
}

\author{
Janusz Telega *, Ryszard Szwaba $@$ and Piotr Doerffer \\ Institute of Fluid Flow Machinery, Polish Academy of Sciences, 80-231 Gdansk, Poland; rssz@imp.gda.pl (R.S.); \\ doerffer@imp.gda.pl (P.D.) \\ * Correspondence: januszt@imp.gda.pl; Tel.: +48-697-626-179
}

Received: 31 October 2019; Accepted: 2 December 2019; Published: 4 December 2019

\begin{abstract}
The results of the experimental research on the symmetry of supersonic flow in a symmetric convergent-divergent nozzle are presented. The investigations were focused on the fact that for some flow conditions the flow in a precisely symmetric nozzle becomes asymmetric. Starting from a specific value of Mach number, the flow becomes asymmetric in terms of shock wave $\lambda$-foot geometry on both sides of a symmetric nozzle. The evolution of the abovementioned asymmetry has been analysed for Mach number value ranging from $\mathrm{M}=1.26$ to $\mathrm{M}=1.59$ with the nozzle opening angle of up to $6.5^{\circ}$ on each side. The presented results indicate that for the same flow parameters as Mach number and Reynolds number, and for the same geometry of the nozzle, different $\lambda$-foot size is formed at each wall. This unexpected behaviour is responsible for the flow asymmetry. Numerical simulations carried out earlier confirm the appearance of shock wave asymmetry. The side in which the asymmetry takes place is accidental, as the full symmetry of simulation mesh and experiment setup was secured. In numerical simulation the asymmetry follows always the same direction. In experiments the direction of asymmetry happens alternatively without any apparent reason. The explanation of the phenomena is provided in this paper.
\end{abstract}

Keywords: $\lambda$-foot; supersonic flow; flow asymmetry; shock waves topology

\section{Introduction}

The phenomena of shock wave asymmetry, in which the $\lambda$-foot on one wall of the nozzle is of a different size than on the other wall is very common in the field of supersonic internal flows. By now it is well established that the height of $\lambda$-foot is influenced by the Reynolds number [1,2], Mach number [3] and the characteristic length scale of boundary layer thickness [4]. In the internal flows, when the shock wave-boundary layer interaction (SWBLI) is constrained by the nozzle there is an additional characteristic feature which is the channel height. The generation of $\lambda$-foot asymmetry in a non-symmetric nozzle [5] is explained by the fact that flow parameters are different at each nozzle wall [6]. An example is presented in Figure 1a schlieren visualization of flow in the very widely exploited experimental case of a 'half-nozzle' with flat bottom wall [7]. In this case, as the nozzle is not symmetric, the above parameters are different on the top and bottom wall giving fairly clear reasons for the asymmetry to arise.

The phenomenon of $\lambda$-foot has been known and widely investigated for over forty years now [8-12], and still draws a lot of attention $[13,14]$. The knowledge of some aspects of this phenomena is increasing. The authors of [15] report the conditions promoting asymmetry and factors driving to this. The authors of [16-18] report that the location of the largest lambda shock of the asymmetric pattern can be forced either on the top, or on the bottom wall, by means of roughness placed on the wall in the throat region.

Also in the field of CFD a lot of work is being done, ranging from the basic general approaches [19] to the more detailed ones. The authors of [20] propose a model based on the properties of fluid entrainment in the mixing layer and momentum conservation, concluding that the entrainment of 
shear layer on the separation is one of the reasons for the asymmetry in the confined SBLI. One of the major problems of CFD still awaiting solution is that in the CFD results the side (top or bottom nozzle wall) that the larger foot occupies remains unchanged for repeated 'flows'. The experiments [20] and author of this paper clearly show, that in experiment the asymmetry can 'flip' between the flows [20]. Yet, the phenomenon of flow asymmetry in symmetric nozzle is still waiting for a full explanation and development of appropriate numerical tools [21]. Figure $1 \mathrm{~b}$ illustrates a schlieren visualization of flow in a fully, symmetric nozzle with different $\lambda$-foot size at each nozzle wall.

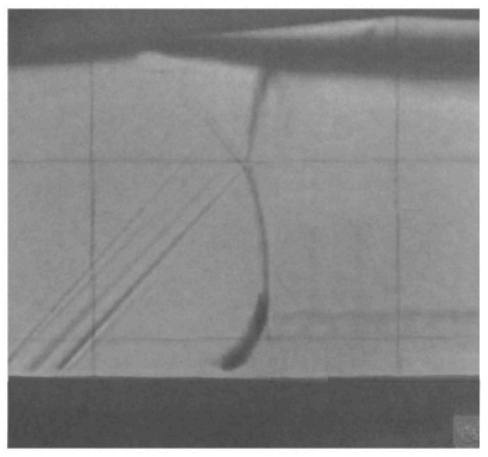

(a)

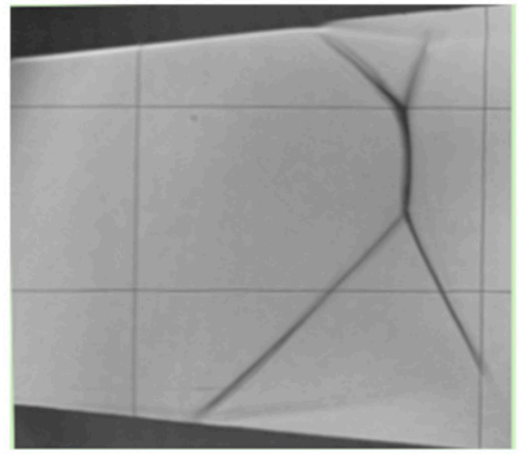

(b)

Figure 1. Shock wave asymmetry in nozzle flows. (a) Asymmetric nozzle with flat bottom wall (b) Symmetric nozzle.

In spite of the nozzle symmetry the asymmetry of flow structure is clear for this case in Figure $1 \mathrm{~b}$. The understanding of reasons behind this counterintuitive asymmetry of the $\lambda$-foot system in a symmetric nozzle is still very low [22]. The asymmetry causes different pressure distribution on the top and bottom wall of a nozzle. This generates a transversal force exerted on the nozzle by the flow. The existence of this component can have very severe consequences especially for a space vehicle. For a typical convergent-divergent propelling nozzle, the conditions promoting asymmetry are present especially during the take-off stage. At this stage, the nozzle is facing the highest possible pressure at the outlet, and the shock is located deep inside the nozzle [23], as depicted in Figure 2.

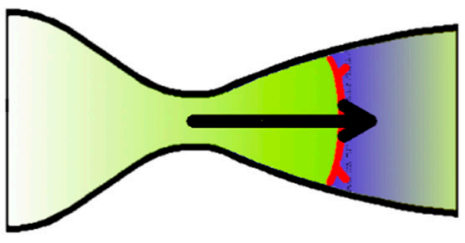

(a)

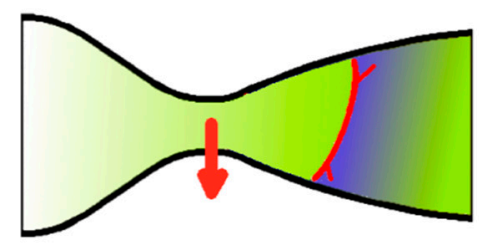

(b)

Figure 2. Thrust force in supersonic nozzle. (a) Symmetric shock system (b) Asymmetric shock system.

The solid black arrow represents flow in the symmetric case. The red arrow represents the presence of side force in the asymmetric case. In Figure 2a the shock system is symmetric thusly the generated thrust is axial (with zero transverse component). On the contrary, Figure $2 b$ illustrates the situation of asymmetric shock system. The lack of symmetry results in different pressure distributions on the top and bottom wall of the nozzle, creating a non-zero traverse component of the net force (marked with red arrow). This force can cause severe consequences during the take-off stage of a space vehicle.

In respect to various nozzle shapes and flow patterns of the shock wave, a general classification of restricted shock separation (RSS) and free shock separation (FSS) is broadly recognized in nozzle flows. It is based on the behaviour of shock-induced boundary layer separation. If the separation is in 
a form of a bubble, restricted by reattachment line still inside the nozzle then it is a RSS. If there is no reattachment inside the nozzle and the separation extends outside the nozzle, one considers the flow as FSS. It is known that side loads resulting from FSS are significantly greater than those generated by RSS [24]. The extreme values of the side force are also reported to accompany the transition from FSS to RSS [23], but this is not the case of our considerations now. We are looking into the asymmetry of $\lambda$-shock system in a quasi-steady state RSS type flow. In our experiments the flow was always an RSS type. The attempt to explain the counterintuitive asymmetry of the $\lambda$-shock system is the main topic of the paper hereafter.

\section{Experimental Setup}

The measurements were carried out at the IMP PAN supersonic wind tunnel facility. The wind tunnel is an intermittent, vacuum type wind tunnel, described in more detailed manner in [25]. The test section with a nozzle is schematically depicted in Figure 3.

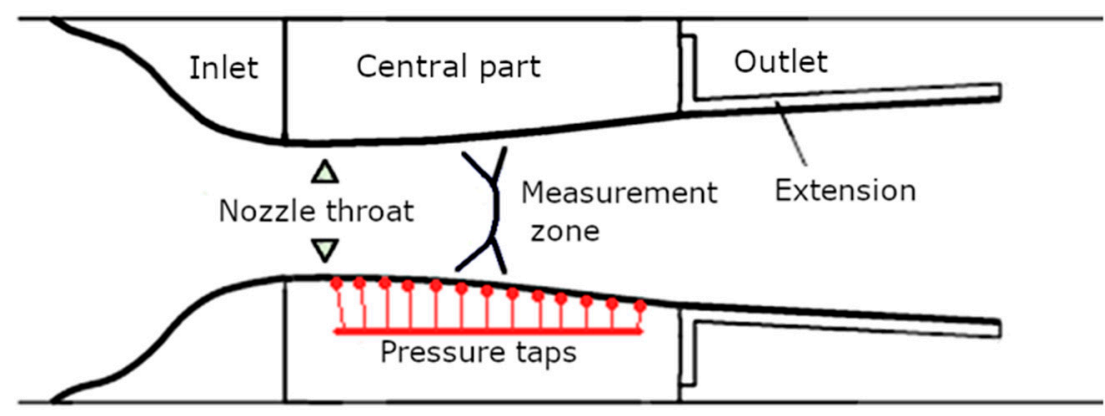

Figure 3. Test section.

The nozzle is composed of carefully machined and mounted identical top and bottom walls. Each of them consists of three elements indicated in the sketch in Figure 3: the inlet part, the central part and the outlet part. The shape of the nozzle central part is a spline followed by a straight line. The nozzle part with rectilinear walls is the test region where the investigated shock waves were located. The outlet part is also rectilinear and in the present measurements its inclination was the same as in the central part. Therefore the walls were straight from the distance upstream of the shock wave to the test section outlet. As the research goal was focusing on asymmetry of the flow in the symmetric nozzle, the process of machining the elements and installing them in the test section had been given a lot of attention. The elements were machined with precision of $0.05 \mathrm{~mm}$ for all linear dimensions. Individually grinded metal pads were used during installation to compensate the imperfections of the test section. The measurements have shown that after installation of the nozzle in the test section, the precision of shape is $0.05 \mathrm{~mm}$ in the no-flow and constant temperature state. We estimate that during the flow the precision is reduced to $0.15 \mathrm{~mm}$ because of the temperature and pressure variations. This number related to the order of magnitude of the macroscopic nozzle dimensions (100 $\mathrm{mm}$ ) yields $0.15 \%$ maximum error of nozzle shape. One can freely and independently change the angles of divergence of these walls. The divergence angle $\delta$ is measured with respect to the symmetry line of the nozzle. It follows that the value of $\delta=6.54^{\circ}$, means that the angle between the nozzle walls is $13.08^{\circ}$. Downstream of the nozzle outlet a control nozzle is installed for the adjustment of the shock location and simultaneously the value of Mach number upstream of the shock wave.

The stagnation temperature upstream of the inlet to the test chamber was measured with PT100 probe thermometer with $0.1{ }^{\circ} \mathrm{C}$ precision. The ambient pressure was measured with Druck DPI 141 manometer, inlet stagnation and static pressure were measured with a Prandtl probe. The distribution of static pressure was measured along the central line of the bottom wall (the pressure taps are marked in Figure 3) of the nozzle with a 64 channel PSI 9010 pressure scanner. First pressure tap was located $50 \mathrm{~mm}$ downstream of the throat followed by 8 taps spaced at intervals of $8 \mathrm{~mm}$. Subsequent 36 taps were spaced regularly every $4 \mathrm{~mm}$. Further 6 taps were spaced evenly every $8 \mathrm{~mm}$ 
and the last one was another $12.35 \mathrm{~mm}$ downstream, being $10 \mathrm{~mm}$ upstream of the outlet part of the nozzle. Altogether 51 taps delivered the streamwise static pressure distribution along the nozzle in the shock interaction area.

For obtaining the topological structure of the flow, schlieren visualisation and Mach-Zhender interferometer visualisation techniques were used. Both of the systems are very 'classical' [26-31] and therefore these are not described in more details here.

\section{CFD Simulations}

As an addendum to the experimental results, also a CFD analysis was taken into account and included besides the experimental results. The results of CFD are based on former publication from our Institute [32-35] and are cited here to show that CFD supports the obtained asymmetry. The CFD analysis was made using the SPARC (Structure Parallel Research Code) [32] solver developed in the 1990s with the cooperation of our group with partners from Karlsruhe University. Presented CFD results are obtained after mesh independency study. To obtain high mesh resolution in the area of shock $\lambda$-foot structure the simulation domain was reduced to the supersonic (divergent) nozzle part. The supersonic inlet condition was obtained from numerous full nozzle simulations, from which the particular inlet velocity profile downstream the nozzle throat was extracted; it included also a turbulent boundary layer profile on side walls. As for the outlet condition, it was assured that the outlet plane of constant static pressure is sufficiently far from the domain of interest. The detailed information on CFD is not given here as for the scope of this paper the CFD is only supplementary to experimental results.

The paper [34,35] covers numerical simulations of flow in symmetric nozzle of divergence angle in the range from $2^{\circ}$ to $6^{\circ}$ and Mach number upstream the shock values from 1.1 to 1.41 . On the basis of numerical simulations the authors conclude that in a symmetric nozzle the flow changes to asymmetric beyond some value of divergence angle and Mach number, like in Figure 4. It is interesting that in the chosen flow configuration (Figure 4) the asymmetry develops along one side of the nozzle.

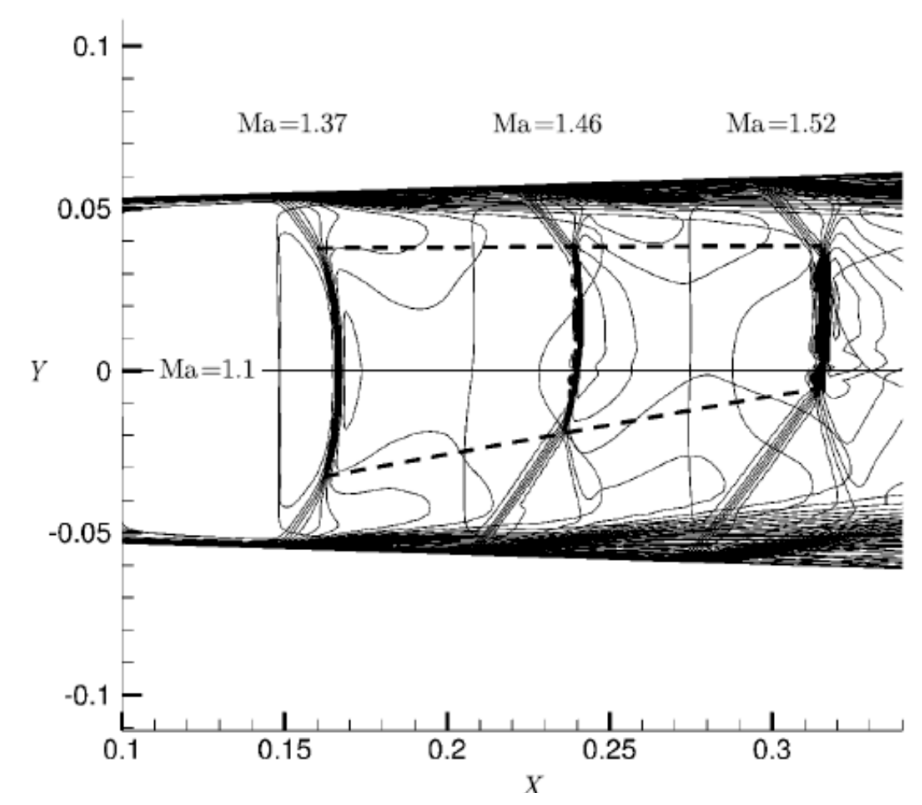

Figure 4. Nozzle divergence angle of $1.877^{\circ}$, inlet $\mathrm{Ma}=1.1$ [34]. Reprint with permission of authors of [34] who hold the copyrights.

Based on the numerical results, the authors of [34] give the concept of the 'division point', that we use now in our present paper for the analysis of experimental results. They also comment on the fact that in CFD results the appearing asymmetry is always in the same direction. This is an effect of small numerical aspects, truncation errors or approximations, which in numerical simulations are 
deterministic. The authors of [34] also show that changing the vertical axis orientation changes the direction of asymmetry.

\section{Measurement Cases}

The measurement cases discussed in this paper are defined in terms of Mach number upstream the shock and divergence angle $\delta$ of the nozzle. The Mach number in experimental cases was calculated from the pressure at the bottom wall. As it has been stated earlier, the divergence angle $\delta$ is measured with respect to the symmetry line of the nozzle. The cases selected for this paper are collected in Table 1.

Table 1. Measurement cases.

\begin{tabular}{cccc}
\hline $\mathbf{M ~ ( - )}$ & $\delta\left(^{\circ}\right)$ & Experimental & CFD \\
\hline 1.31 & 4.5 & $\sqrt{ }$ & $\mathrm{X}$ \\
1.32 & 6.54 & $\sqrt{ }$ & $\sqrt{ }$ \\
1.36 & 3 & $\sqrt{ }$ & $\mathrm{X}$ \\
1.36 & 4.5 & $\sqrt{ }$ & $\mathrm{X}$ \\
1.40 & 3 & $\sqrt{ }$ & $\mathrm{X}$ \\
1.40 & 4.5 & $\sqrt{ }$ & $\mathrm{X}$ \\
1.45 & 4.5 & $\sqrt{ }$ & $\mathrm{X}$ \\
1.57 & 6.54 & $\sqrt{ }$ & $\mathrm{X}$ \\
1.59 & 6.54 & $\mathrm{X}$ & $\sqrt{ }$ \\
\hline
\end{tabular}

The experimental cases cover Mach number range from $\mathrm{M}=1.31$ to $\mathrm{M}=1.57$. It should be pointed out that additional numerical results are very few and serve as confirmation of asymmetry existence. Only two cases have been simulated numerically, to illustrate the coincidence of results.

\section{Results}

Measured pressure distributions in terms of isentropic Mach number on the bottom wall of the nozzle are presented in Figure 5 for four chosen cases.

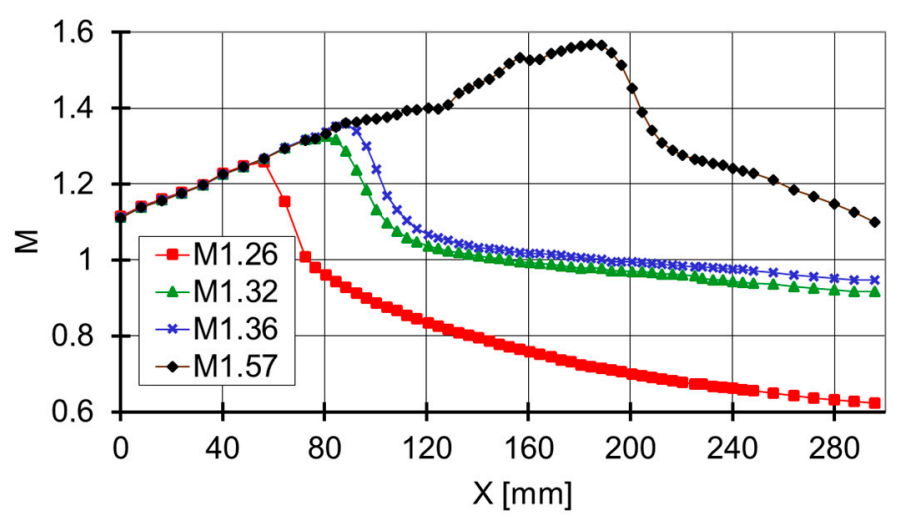

Figure 5. Mach number distribution on the bottom wall.

Prior to the shock wave location, all the points follow exactly the same curve, resulting from the nozzle shape. Shock wave presence causes significant Mach number drop. Its location does not display any influence on the Mach number distribution upstream the shock wave. Downstream the shock location a further deceleration towards the nozzle exit takes place.

Schlieren photographs (Figure 6a) and interferograms (Figure 6b) were the backbone for the investigation of flow topology of the system. The height of the top $\lambda$-foot ' $h 1$ ' and the height of bottom $\lambda$-foot ' $\mathrm{h} 2$ ' are illustrated in Figure 6a. 


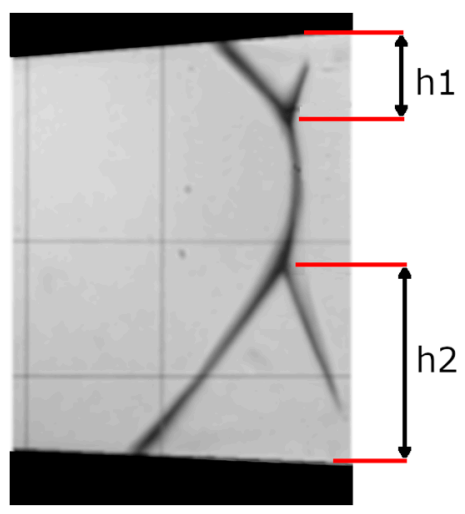

(a)

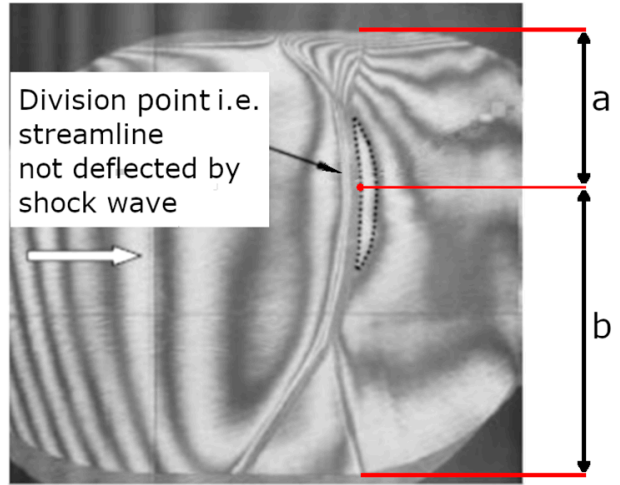

(b)

Figure 6. The $\lambda$-feet geometrical parameters definition. (a) Schlieren image. (b) Interferogram.

In order to parametrize the shock waves topology a new term, the 'division point' was introduced. It is defined as the position of the point on a shock wave being perpendicular to the local flow velocity direction. The shock between both triple points is oblique but of the strong type, so it looks nearly normal all over. But it is normal only in one point between triple points. In this particular point the shock is the strongest and therefore the density jump is maximal. Downstream the shock wave therefore, a maximum of density is built-up which is indicated by the 'looped' fringe line. This closed line is stretched along the shock because of weak variation of the shock intensity. The centre of this closed fringe line is the place where the dividing streamline is passing through. In the symmetric flow case this line is the geometrical central line of the nozzle.

Analogical parameters were determined on the basis of numerical results from Mach number contours, presented in Figure 7.

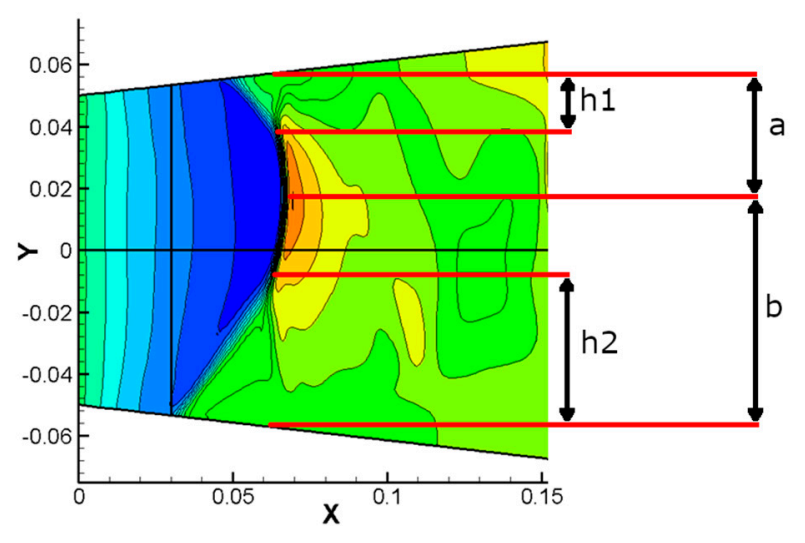

Figure 7. The $\lambda$-feet parameters marked on numerical results.

The 'normalised heights' (' $\mathrm{q}$ ' - ratios of shock wave triple point height and corresponding height of the 'division point' position) of the top ' $\mathrm{h} 1 / \mathrm{a}$ ' and bottom ' $\mathrm{h} 2 / \mathrm{b}$ ' $\lambda$-feet are given in Table 2.

The values given in Table 2 show that with error smaller than $1.5 \%$ the normalized height of the top and bottom $\lambda$-feet remain the same also in asymmetric flows. This observation is confirmed in both experimental and CFD results. The transition from symmetric to asymmetric flow topology is illustrated in Table 2 in terms of the size of normalized $\lambda$-feet ' $q$ ' as a function of Mach number upstream the shock wave. 
Table 2. Normalized height.

\begin{tabular}{|c|c|c|c|c|c|c|c|c|c|}
\hline \multirow[t]{2}{*}{ M (-) } & \multirow[t]{2}{*}{$\delta\left({ }^{\circ}\right)$} & \multicolumn{4}{|c|}{ Experimental } & \multicolumn{4}{|c|}{ CFD } \\
\hline & & h1/a & h2/b & Flow & Diff. & h1/a & h2/b & Flow & Diff. \\
\hline 1.31 & 4.5 & \multicolumn{2}{|c|}{0.3187} & Symmetric & & & & & \\
\hline 1.32 & 6.54 & 0.615 & 0.622 & Asymmetric & $1.1 \%$ & 0.531 & 0.524 & Asymn & c $1.3 \%$ \\
\hline 1.36 & 3 & \multicolumn{2}{|c|}{0.4611} & Symmetric & & & & & \\
\hline 1.36 & 4.5 & 0.64 & 0.632 & Asymmetric & $1.3 \%$ & & & & \\
\hline 1.40 & 3 & \multicolumn{2}{|c|}{0.5075} & Symmetric & & & & & \\
\hline 1.40 & 4.5 & 0.6377 & 0.6377 & Asymmetric & $0 \%$ & & & & \\
\hline 1.45 & 4.5 & 0.6451 & 0.6451 & Asymmetric & $0 \%$ & & & & \\
\hline 1.57 & 6.54 & 0.763 & 0.767 & Asymmetric & $0.5 \%$ & & & & \\
\hline 1.59 & 6.54 & & & & & 0.795 & 0.798 & Asymn & c $0.4 \%$ \\
\hline
\end{tabular}

Each curve in Figure 8 corresponds to a different divergence angle of the nozzle. The shape of the marker indicates the presence of the symmetry. Circle means a symmetric flow, while the triangle denotes the asymmetric one. There is a clear tendency that the symmetry breaks as the Mach number upstream the shock increases, and also when the angle of nozzle divergence increases. If these two are sufficiently high, the asymmetry is likely to appear. For adequately high nozzle divergence angle the symmetry tends to break when the reversed flow caused by the separation becomes noticeable. At this point this complex system of flow with shock waves is looking for a new stable topology.

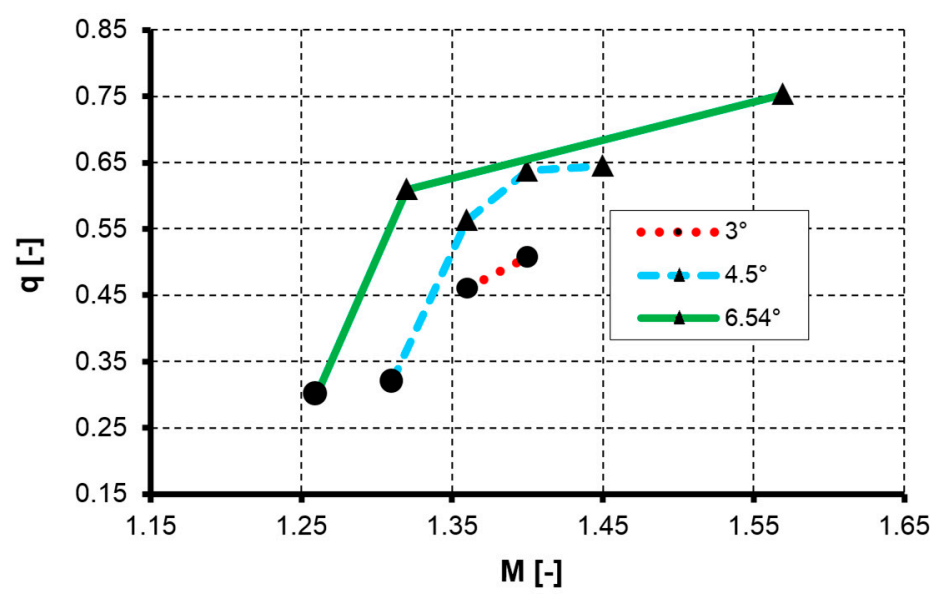

Figure 8. Normalized height and the zones of symmetry for divergence angle values of $3^{\circ}, 4.5^{\circ}, 6.54^{\circ}$.

\section{Discussion}

The existence of an asymmetric system in a symmetric flow is very confusing and counter-intuitive. One would expect that the top and bottom shock systems being mirror copies with both $\lambda$-feet exactly of the same height and the streamline of no deflection (normal shock) located in the axis of the nozzle $\left(' h 1^{\prime}={ }^{\prime} h 2^{\prime}\right.$ and ' $\left.a{ }^{\prime}={ }^{\prime} b '\right)$. It is illustrated schematically in Figure 9a. This is true only up to a certain Mach number value. 


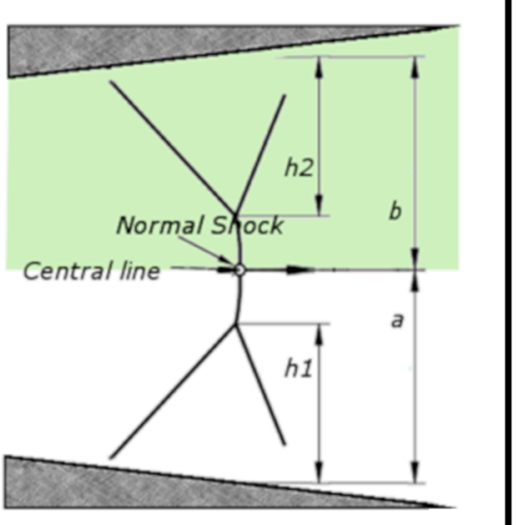

(a)

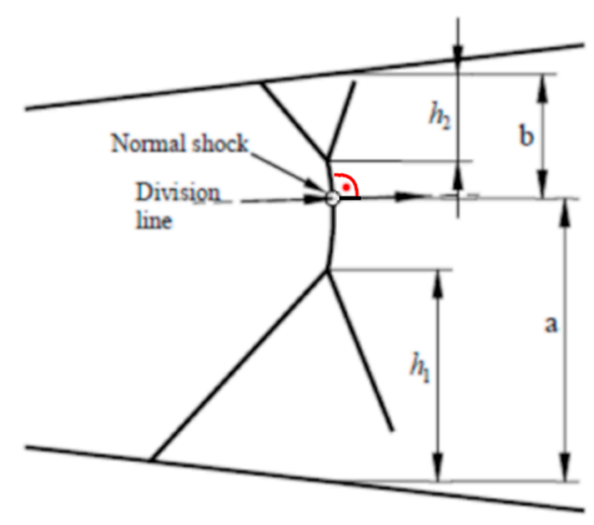

(b)

Figure 9. Symmetrical and asymmetrical shock wave. (a) Symmetric shock system. (b) Asymmetric shock system.

In the carried out experiments the flow is symmetric for low Mach number and low $\delta$ angle. However, for higher Mach number and $\delta$ angle values, the flow is not symmetric any more. The flow pattern changes to one depicted in Figure $9 \mathrm{~b}$. In asymmetric cases the $\lambda$-feet height and distance from wall to the division line are different for the top and the bottom wall ('a' $\neq{ }^{\prime} \mathrm{b}{ }^{\prime}$ and ' $\left.h 1^{\prime} \neq{ }^{\prime} \mathrm{h} 2^{\prime}\right)$. The break of symmetry can be caused by either increase of Mach number or by increase of nozzle divergence angle. As the number of cases considered here is relatively low, further research is required to give a more precise insight of the influence of Mach number and divergence angle on the extent of symmetry zone.

On the basis of the results presented, we put forward a hypothesis that after topological symmetry of the flow breaks, the 'normalised' symmetry of $\lambda$-feet remains in the flow. The 'physical' understanding of symmetry expressed by normalising the height of $\lambda$-feet by the position of the division line takes into account not just geometrical size of the $\lambda$-feet but also the topology of the flow. We claim and have proven that the system of $\lambda$-feet is symmetric with reference to the position of normal shock wave position marked as 'division line' in Figure 9b. This 'division line' is the streamline perpendicular to the shock wave. In symmetric case this is the geometrical centre line of the nozzle. In the case when geometrical symmetry of the flow is broken, this line is shifted towards one of the nozzle walls: either top or bottom one. This line fixes the heights of two parts that the flow is divided into. In internal flows the height of the channel is a significant factor influencing the size of the $\lambda$-foot because the shock wave-boundary layer interaction is constrained in a nozzle.

\section{Conclusions}

The results given in the paper prove experimentally that if the sizes of $\lambda$-feet are measured in ratio to the height of division point, than this 'normalised size' of both of them is the same. This means that despite geometrical asymmetry, the flow remains symmetric if the dimensions are related to the position of the new division line that is generated spontaneously in the flow and it is not the geometrical central line of the nozzle. The streamline that is perpendicular to the shock wave defines the division line of the flow. The position of this streamline is the constraint dividing the flow. This explains the behaviour of the flow, as it is the only parameter that is not the same for the top and bottom $\lambda$-foot in a symmetric nozzle. The above behaviour is the characteristic feature of the $\lambda$-foot formation in the constrained environment.

Author Contributions: Conceptualization, P.D.; data curation, R.S.; formal analysis, J.T.; investigation, J.T. and R.S.; methodology, R.S.; supervision, P.D.; writing—original draft, J.T.; writing—review and editing, R.S. and P.D.

Funding: This research received no external funding.

Conflicts of Interest: The authors declare no conflict of interest. 


\section{References}

1. Doerffer, P.; Zierep, J. An experimental investigation of the Reynolds number effect on a normal shock wave-Turbulent boundary layer interactions on a curved wall. Acta Mech. 1988, 73, 77-93. [CrossRef]

2. Doerffer, P.; Dallmann, U. Reynolds number effect on separation structures in normal shock wave/turbulent boundary-layer interaction. AIAA 1989, 27, 9. [CrossRef]

3. Doerffer, P. An experimental investigation of the Mach number effect upon a normal shock wave/Turbulent boundary layer interaction on a curved wall. Acta Mech. 1989, 76, 35-51. [CrossRef]

4. Doerffer, P. Normal shock $\lambda$-foot topography at turbulent boundary layer. Acta Mech. 1994, 4, $133-140$.

5. Doerffer, P.; Dallmann, U. Mach number dependence of flow separation induced by normal shock-wave/turbulent boundary-layer interaction at a curved wall. In Proceedings of the AIAA conference, Reno, NV, USA, 9-12 January 1989.

6. Doerffer, P.; Dallmann, U. Separation structures produced by normal shock wave/turbulent boundary layer interaction in a narrow wind tunnel. In Proceedings of the AIAA Conference, Honolulu, HI, USA, 8-10 June 1987.

7. Szwaba, R. Shock wave induced separation control by streamwise vortices. J. Therm. Sci. 2005, 14, 249. [CrossRef]

8. Doerffer, P.; Dallmann, U. Spatial and temporal features of a separated flow field at a convex wall induced by normal shock wave turbulent boundary layer interaction. AIAA Pap. 1999, 90, 457.

9. Ackeret, J.; Feldman, F.; Rott, H. Unterrsuchungen an VerdichtungsstoSen und Grenzschichten in Schell Bewegten Gasen; Bericth des Institutes fur Aerodynamik, ETH Zurich: Zurich, Switzerland, 1946; Volume 10.

10. Seddon, J. The Flow Produced by Interaction of Turbulent Boundary Layer with a Normal Shock Wave of Strength Sufficient to Cause Separation; ARC R\&M: London, UK, 1967; p. 3502.

11. Lawrence, R.A. Symmetrical and Unsymmetrical Separation in Supersonic Nozzles; Research report 67-1; Southern Methodist University: Dallas, TX, USA, 1967.

12. Thompson, R.L. A Study of the Effects Produced by Asymmetrical Nozzle Entrance Geometries on the Downstream Supersonic Flow; Air Force Institute of Technology: Wright-Patterson AFB, OH, USA, 1968.

13. Golub, V.V.; Efremov, S.V.; Saveliev, A.S. Asymmetric Flow Separation in de Laval Nozzle. In 28th International Symposium on Shock Waves; Kontis, K., Ed.; Springer: Berlin/Heidelberg, Germany, 2012.

14. Tianyun, G.; Jianhan, L.; Mingbo, S. Symmetric/asymmetric separation transition in a supersonic combustor with single-side expansion. Phys. Fluids 2017, 29, 126102.

15. Verma, S.B.; Manisankar, C. Origin of flow asymmetry in planar nozzles with separation. Shock Waves 2014, 24, 191-200. [CrossRef]

16. Reijasse, P.; Corbel, B.; Soulevant, D. Unsteadiness and asymmetry of shock-induced separation in a planar two-dimensional nozzle: A flow description. In Proceedings of the AIAA Conference, Norfolk, VA, USA, 28 June-1 July 1999.

17. Reijasse, P. Aerodynamical aspects of propulsive nozzle flow in off-design regime. In Proceedings of the $3 \mathrm{rd}$ European Symposium on Aerothermody-namics for Space Vehicles, ESTEC, Noordwijk, The Netherlands, 24-26 November 1998.

18. Bourgoing, A.; Reijasse, P. Analysis of unsteady separated flows in a supersonic planar nozzle. Shock Waves 2005, 14, 251-258. [CrossRef]

19. Czerwińska, J. The Analysis of Separation Methods of Three-dimensional Flow Structure Detection in the Boundary Layer Shock Wave Interaction. Task Q. 1999, 1, 53-140.

20. Wang, C.; Zhuo, C. Model for Asymmetry of Shock/Boundary Layer Interactions in Nozzle Flows. Trans. Nanjing Univ. Aeronaut. Astronaut. 2018, 35, 146-153.

21. Bourgoing, A.; Benay, R. Invesatigation of an Asymmetrical Shock-Wave Boundary-Layer interaction in a Supersonic Planar Nozzle. Can. Aeronaut. Space J. 2005, 51, 71-86. [CrossRef]

22. Papamoschou, D. Supersonic flow separation in planar nozzles. Shock Waves 2008, 19, 171-183. [CrossRef]

23. Matsuo, S.; Suetsugu, S.; Nagao, J.; Hashimoto, T.; Setoguchi, T.; Kim Heuy, D. Control of the asymmetric flow in rocket nozzles. J. Therm. Sci. 2013, 22, 97-102. [CrossRef]

24. Olson, B.; Lele, S. A mechanism for unsteady separation in over-expanded nozzle flow. Phys. Fluids 2013, 25, 110809. [CrossRef] 
25. Fomin, N.; Lavinskaya, E.; Doerffer, P.; Szumski, J.A.; Szwaba, R.; Telega, J. Quantitative diagnostics of shock wave-boundary layer interaction by digital speckle photography. In Shock Waves, 1st ed.; Hannemann, S.F., Ed.; Springer: Berlin/Heidelberg, Germany, 2009.

26. Settles, G.S. Schlieren and Shadowgraph Techniques, 1st ed.; Springer: Berlin/Heidelberg, Germany, 2001.

27. Smits, A.J.; Lim, T.T. Flow Visualization: Techniques and Examples; Imperial College Press: London, UK, 2000.

28. Merzkirch, W. Flow Visualization, 2nd ed.; Academic Press, Inc.: Cambridge, MA, USA, 1987.

29. Zetie, K.P. How does a Mach-Zehnder interferometer work? Phys. Educ. 2000, 35, 1. [CrossRef]

30. Price, E.W. Initial Adjustment of the Mach-Zehnder Interferometer. Rev. Sci. Instrum. 1952, $23,162$. [CrossRef]

31. Discetti, S.; Ianiro, A. Experimental Aerodynamics, 2nd ed.; CRC Press: Boca Raton, FL, USA, 2017.

32. Mangagnato, F. KAPPA-Karlsruhe parallel program for aerodynamics. Task Q. 1998, 2, 215-270.

33. Szwaba, R.; Doerffer, P.; Namiesnik, K.; Szulc, O. Flow structure in the region of three shock wave interaction. Aerosp. Sci. Technol. 2004, 8, 499-508. [CrossRef]

34. Namieśnik, K.; Doerffer, P. Numerical Simulation of Shock Wave Patterns in Supersonic Divergent Symmetric Nozzles. TASK Q. 2004, 9, 53-63.

35. Szwaba, R.; Namieśnik, K.; Doerffer, P. Powstawanie niesymetrycznego układu fal w dyszy symetrycznej (in Polish). In Proceedings of the Book of Abstracts of KKMP Conference, Waplewo, Poland, 20-23 September 2004.

(C) 2019 by the authors. Licensee MDPI, Basel, Switzerland. This article is an open access article distributed under the terms and conditions of the Creative Commons Attribution (CC BY) license (http://creativecommons.org/licenses/by/4.0/). 